\title{
Necesidad del uso de tesauros \\ en el manejo de documentación administrativa en archivos y centros de documentación
}

\author{
Rosana López Carreño \\ Grupo de Tecnologías de la Información \\ Universidad de Murcia \\ E-mail: Rosana.Lopez@carm.es
}

\subsection{Resumen}

En los últimos años las Administraciones Públicas vienen desarrollando continuos esfuerzos en pos de la modernización, aunque pocas veces se aborda con detenimiento la problemática de la gestión de los documentos administrativos. Con el objeto de promover una normalización de los procedimientos administrativos, introducimos la idea de utilizar el tesauro como instrumento para la gestión de esta documentación, medio que nos permitirá alcanzar dos objetivos fundamentales: una consistente normalización terminológica y, por consiguiente, una adecuada recuperación de la información dentro de un entorno relacional. Presentamos la experiencia desarrollada en el seno del Archivo Forestal de la Dirección General del Medio Natural de la Consejería de Medio Ambiente, Agricultura y Agua de la Comunidad Autónoma de la Región de Murcia, resaltando la singular presencia del empleo de este lenguaje documental en un contexto tan poco común.

Palabras Clave: Tesauros. Archivos. Gestión de información. Medio ambiente. Administraciones públicas.

\subsection{Abstract}

Although public administrations in Spain have been developing continuous efforts during the last years to modernize their structures and procedures, only a few cases exemplifying these activities in the field of administrative archives have been documented. This paper emphasized the role that thesauri can play in this field, incorporating vocabulary control to the description and retrieval of administrative records. The experience developed into the Forest Archive of the Regional Government of Murcia is introduced. Finally, an analysis of interac-

Scire. 5 : 2 (jul.-dic. 1999) 115-130. 
tions established between the natural environment thesaurus and the relational database system —implemented to improve the efficiency and effectiveness in information retrieval- is offered.

Keywords: Thesauri. Archives. Information Management. Natural environment. Public Administrations.

\section{Introducción}

La Ley 30/1992, de 26 de noviembre, de Régimen Jurídico de las Administraciones Públicas y del Procedimiento Administrativo Común, instaura un nuevo concepto de las relaciones informativas entre las Administraciones Públicas y los ciudadanos, postulando la idea de que estos organismos deben proceder a la adaptación de sus normas y procedimientos, utilizando como medio para dicho fin las instituciones documentales que en ellos se integran. Si tenemos presente que las instituciones documentales (Archivos, Bibliotecas, Mediatecas y Centros de Documentación), de las Administraciones Públicas, son los organismos responsables del tratamiento, custodia y recuperación de la información y documentación necesaria para los procesos de tomas de decisiones, no es necesario recordar la importancia de estas instituciones dentro del proceso de modernización de la Administración.

En este trabajo nos centraremos en los archivos y centros de documentación, en los que la materia prima es la documentación administrativa. Basándonos en la idea general de que el archivero o documentalista responsable de la gestión del archivo debe ser un profesional que extienda toda su actividad a la globalidad del proceso de tratamiento documental de los expedientes administrativos, parece claro que es precisamente en el seno del archivo de donde debe emanar el proceso de planificación de la gestión de información y documentación, asumiendo la responsabilidad de diseñar y elaborar un verdadero sistema gestor de la información y documentación administrativa dentro de la institución, favoreciendo un sencillo almacenamiento y una rápida recuperación de los documentos requeridos y, por otro lado, el archivo debe, así mismo, emitir o participar en la redacción de las directrices apropiadas para la puesta en funcionamiento de un verdadero mecanismo de circulación de la información.

Tomamos como base de nuestra propuesta el conjunto global de las teorías archivísticas norteamericanas, que exponen una concepción de Archivo activo y dinámico, constituyéndose éste como un Sistema de Información vital e imprescindible dentro de la institución en la cual está inmerso. Teniendo presente que los documentos deben ser objeto de tratamiento documental, no sólo cuando el emisor se desentiende de ellos, sino incluso desde el momento anterior a la formación del documento, no hace falta recordar la importancia de una buena

Scire. $5: 2$ (jul.-dic. 1999) 115-130. 
Gestión de los Documentos Administrativos (Records Management), como conjunto de tareas y procedimientos orientados a obtener una mayor eficacia y economía en la explotación de los documentos por parte de las administraciones, permitiendo alcanzar así una óptima Gestión del Sistema de Archivos General de la institución.

Con nuestro trabajo intentamos reflejar la problemática general que comienza a plantearse en el tratamiento de la documentación depositada en los archivos administrativos de las Administraciones Públicas, y a un nivel más particular, en el seno de la Consejería de Medio Ambiente, Agricultura y Agua de la Comunidad Autónoma de la Región de Murcia, debido a la endémica carencia de tratamiento documental que se ha venido arrastrando a lo largo del tiempo en esta instiución, y que ha venido provocando un caos informativo y documental total, aumentado más si cabe, con la introducción de las Tecnologías de la Información, las cuales están provocando un crecimiento exponencial de la documentación administrativa.

Ante esta situación, debemos considerar que si los documentos administrativos son el soporte en el que se materializan los distintos actos de la Administración Pública, la Gestión de la Documentación Administrativa se constituye como una pieza fundamental en el puzzle de dicha Administración. Esbozamos la idea de que el inicio de la planificación de dicha gestión tiene que transcurrir, inevitablemente por el establecimiento de una serie de etapas primordiales como son: el estudio funcional y orgánico de la institución, el ciclo de vida, el tratamiento y custodia de la documentación, así como su automatización y posterior difusión de la misma.

Centrándonos en este último paso, que sería el punto donde culminaría el proceso de Modernización del Sistema de Información Administrativo, debemos señalar la necesidad de adoptar una normalización conceptual y terminológica, ya que se considera a éste como un proceso que afecta directamente a la gestión documental y que nos permite paliar la falta de precisión terminológica y la confusión conceptual. Junto con los instrumentos de descripción utilizados de forma tradicional (guías, catálogos e inventarios propios de los archivos), consideramos que debemos añadir la presencia de los lenguajes documentales, que además de permitir un control en el tratamiento de los fondos y dotar de una cierta normalización al proceso, van a aportar, con su uso, una novedad importante dentro de la gestión de la documentación administrativa y archivística. Dentro de estos lenguajes de naturaleza controlada, para nosotros se constituirá el Tesauro como una herramienta enormemente valiosa para alcanzar los objetivos propuestos en el proceso de Normalización. 


\section{8}

Susana López Carreño

\section{Metodología}

Un precedente del uso del Tesauro como herramienta archivística lo encontramos en el caso de los Archivos del Gobierno Australiano (Office for government online) que vienen trabajando en la idea de elaborar un Tesauro común de términos administrativos, por medio de la colaboración entre los distintos organismos que integran dicha administración, considerando al igual que nosotros, que el uso de un tesauro para la gestión de archivos reducirá significativamente el esfuerzo de normalización administrativa y mejorará el acceso a la información y documentación de los archivos, ante el aumento continuado del volumen de la información gestionado por las distintas agencias del gobierno. Para ello se propugna la incorporación de sistemas que permitan la recuperación de información en lenguaje natural, que precisan del despliegue previo de un tesauro acompañado, posteriormente de la implementación de distintas herramientas de búsqueda basadas en técnicas de Inteligencia Artificial.

Este proyecto de la Administración australiana se desarrolla con la idea de conseguir un aumento de la visibilidad de los fondos custodiados por el Estado y se ha aprobado, en un principio el establecimiento de un sistema de identificación de la información, AusGILS (Australian Government Information Locator System) y se ha solicitado a los Archivos Australianos el desarrollo de un plan de implementación de forma urgente y prioritaria. También se ha recomendado la reutilización, en tanto sea posible, de trabajos similares llevados a cabo en los Estados Unidos de Norteamérica (Government Information Locator Service). En el caso australiano, se ha procedido a la puesta en funcionamiento de un foro electrónico de debate (thesaurus@dpie.gov.au) destinado al intercambio de información sobre la creación, mantenimiento y uso de los tesauros y sistemas de clasificación entre los distintos organismos del gobierno australiano y agencias de la Commonwealth.

Los norteamericanos promueven el concepto de GILS con la idea de beneficiar a cualquier usuario que precise algún tipo de información de la Administración Federal o a los propios funcionarios encargados de recuperar información para los ciudadanos. Se pretende que las operaciones de búsqueda de información se realicen directamente a través de cualquier estación de trabajo conectada a Internet o cualquier otro tipo de red. Un registro GILS nos proporciona descripciones de la información pública disponible recogiendo un minucioso detalle de la fuente de información, incluyendo qué información podemos encontrar, cuándo fue creada y puesta a disposición de los usuarios, cómo contactar para más información y, en algunos casos, se aporta un enlace directo con los administradores del sistema. 
En similar línea de trabajo, conducente a introducir un formato común para la identificación y descripción de la información administrativa, viene trabajando la administración canadiense (What is GILS?); que como puede apreciarse en las siguientes figuras, el Tesauro se considera un instrumento fundamental, pero no sólo en este proceso de identificación y descripción sino también en la recuperación de información tanto interna como externa a la administración.

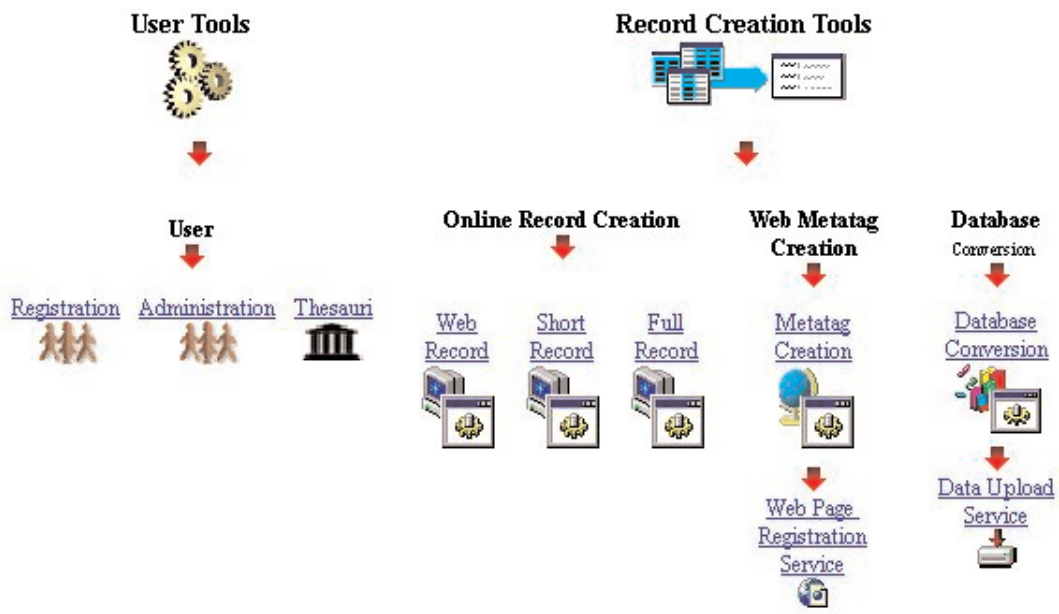

Fig. 1. El Tesauro como herramienta de normalización dentro de la Administración Canadiense

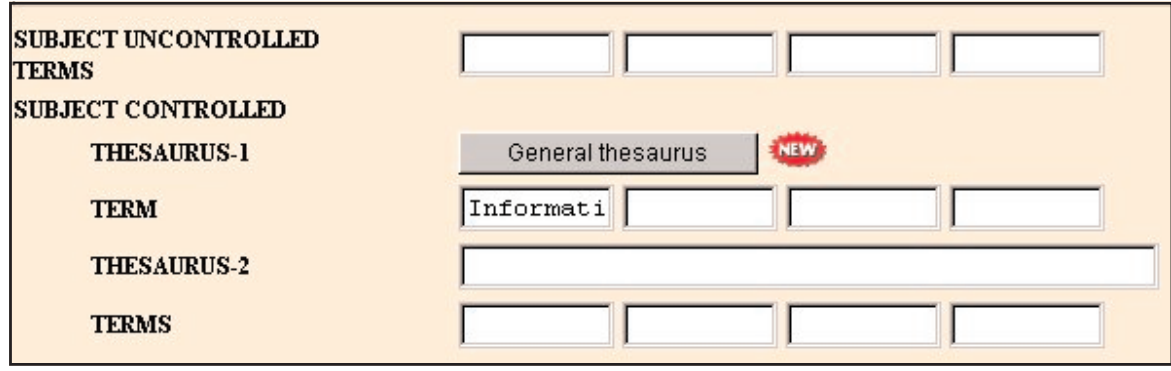

Fig. 2 Sección del Formulario de Introducción de Nuevo Registro en el sistema GLIS canadiense

Scire. 5 : 2 (jul.-dic. 1999) 115-130. 
Para resaltar nuestra creencia en la utilidad de los lenguajes documentales y más concretamente del tesauro, dentro de la gestión de la documentación administrativa en las Administraciones Públicas, este trabajo se va a ejemplificar con la experiencia profesional que llevamos a cabo en este tema, en el seno del Archivo Forestal de la Consejería de Medio Ambiente, Agricultura y Agua, de la Comunidad Autónoma de la Región de Murcia.

El Tesauro del Archivo Forestal es uno de los frutos obtenidos del trabajo que se viene desempeñando en dicho Archivo desde el año 1997, actividad que surge cuando en la Consejería se constata la perentoria necesidad de una adecuada organización, tratamiento y automatización de la documentación que allí se hallaba depositada.

Ante la situación caótica que acontecía en el Archivo Forestal, se procedió a su organización, pero, como paso previo e imprescindible se ha debido realizar un profundo estudio sobre el origen y evolución de su organismo emisor, en este caso la Dirección General del Medio Natural de la Consejería de Medio Ambiente, Agricultura y Agua. Este estudio se hizo con detenimiento ya que se presentaban diversos problemas, entre los que destacaban la gran diversidad de tipos documentales y sobre todo la redundancia, sinonimia y variedad terminológica en la descripción y nomenclatura de los tipos documentales pertenecientes a este archivo, lo cual nos llevó a considerar necesario el uso de un lenguaje controlado para poder establecer una mejor gestión de dicha documentación. Por todo ello, hay que hacer una breve referencia a los cambios estructurales y funcionales sufridos por el organismo emisor y que afectan directamente a estos tipos documentales, ya que hay que tener en cuenta que se trata de un archivo de oficina e intermedio a la vez.

\subsection{Estudio Histórico del Organismo Emisor}

A pesar de que las primeras normas protectoras de los bosques aparecen en la Baja Edad Media (Parada, 1993), nos centraremos en la segunda mitad de este siglo, que es donde la Administración Forestal resultó muy transformada y donde ya hay relación con algunos de los tipos documentales de este Archivo.

\subsection{1. Ámbito Nacional}

La fuerte intervención administrativa se inició con la Ley de 10 de marzo de 1941, que creó el Patrimonio Forestal del Estado. El Sistema Forestal vigente se establece a partir de la Ley de Montes, actualmente vigente, de 8 de junio de 1957, junto con su Reglamento, aprobado por Decreto de 22 de febrero de 1962. En 1971 se crea el Instituto Nacional para la Conservación de la Naturaleza (ICONA), como organismo autónomo de carácter comercial, industrial y financiero.

Scire. $5: 2$ (jul.-dic. 1999) 115-130. 
En 1991 se establece la estructura orgánica básica del Ministerio de Agricultura Pesca y Alimentación y dentro de su Secretaría General de Estructuras Agrarias, se incluye el Instituto Nacional para la Conservación de la Naturaleza (ICONA), que se compone de un Director General, Secretaría General, Subdirección General de Protección de la Naturaleza, Subdirección General de Espacios Naturales y Subdirección General de Ordenación de Recursos Naturales. Y en 1995 se modifica parcialmente la estructura orgánica básica del Ministerio de Agricultura, Pesca y Alimentación. Y el Instituto Nacional para la Conservación de la Naturaleza (ICONA), se refunde con el Instituto Nacional de Reforma y Desarrollo Agrario (IRYDA), pasando a denominarse Parques Naturales. Se crea Parques Naturales como organismo autónomo y se establecen sus funciones, dependiente actualmente del Ministerio de Medio Ambiente.

La Estructura Orgánica actual del Ministerio de Medio Ambiente, se divide fundamentalmente en: la Secretaría de Estado de Aguas y Costas, la Secretaría General de Medio Ambiente, dentro del cual se encuentra la Dirección General de Conservación de la Naturaleza, del que depende el Organismo Autónomo Parques Nacionales; y la Subsecretaría de Medio Ambiente.

\subsection{2. Ámbito Autonómico}

El cambio más significativo fue provocado por la incidencia del Estado de las Autonomías, que supuso prácticamente la desaparición de la Administración Estatal a favor de otras tantas Administraciones de las Comunidades Autónomas. En el Real Decreto 2102/1984, de 10 de octubre, se traspasan las funciones y servicios del Estado a la Región de Murcia en materia de conservación de la naturaleza.

La Ley del 19-12-1986, núm. 10/1986, crea la Agencia Regional para Medio Ambiente y la Naturaleza de la Región de Murcia (ARMAN), como organismo autónomo de carácter administrativo adscrito a la Presidencia de la Comunidad Autónoma. Y en la Ley del 5-11-1993, núm. 6/1993, se suprime la Agencia Regional para Medio Ambiente y Naturaleza de la Región de Murcia. El Consejo Asesor Regional de Medio Ambiente se adscribe a la Consejería de Medio Ambiente. En el Decreto 41/1994, de 8 de abril, se establece la Estructura Orgánica de la Consejería de Medio Ambiente. Y en el Real Decreto 647/1995, del 21 de abril, se amplían los medios adscritos a los servicios a los servicios traspasados a la Comunidad Autónoma de la Región de Murcia, en materia de conservación de la naturaleza.

En 1995, se fusionan, la Consejería de Medio Ambiente con la Consejería de Agricultura, Ganadería y Pesca ya existente y cuya última modificación en su estructura orgánica fue mediante fue mediante el Decreto 73/1992, de 2 de julio.

Scire. $5: 2$ (jul.-dic. 1999) 115-130. 
Así se constituye la Consejería de Medio Ambiente, Agricultura y Agua y mediante el Decreto 63/1996, de 2 de agosto, se establece la Estructura Orgánica, aún vigente. $Y$ aunque ha habido otros cambios, pero que no afectaban directamente a la estructura establecida en materia de Medio Ambiente, excepto la creación de la Secretaría Sectorial de Agua y Medio Ambiente (Decreto 123/1995, de 26 de julio), que junto con las Direcciones Generales del Agua, del Medio Natural y de Protección Civil y Ambiental, gestionan todo lo relacionado en materia de conservación de la naturaleza dentro de la Consejería de Medio Ambiente, Agricultura y Agua.

Una vez que hemos llegado a la estructura orgánica actual de la Consejería de Medio Ambiente, Agricultura y Agua, ésta se encuentra compuesta por Secretaría General, Secretaría Sectorial de Agua y Medio Ambiente y seis Direcciones Generales, la localización dentro de este organigrama, del Archivo Forestal se encuentra bajo la gestión de la Dirección General del Medio Natural.

\subsection{Control del vocabulario}

Tras el estudio y análisis de los cambios estructurales y funcionales del organismo emisor se procedió a la organización del Archivo Forestal y cuando se empezó a clasificar y ordenar las distintas series documentales ya se preveía la necesidad de elaborar una herramienta de control terminológico que nos sirviera de apoyo, no sólo para la organización del fondo sino también en otras etapas tan importantes como la descripción de los tipos documentales, la informatización de los datos obtenidos en la descripción y sobre todo la posterior recuperación de información.

En el progreso de esta etapa, la mayor cantidad de problemas que se nos presentaron, residía en el proceso de la descripción de los tipos documentales, donde detectamos abundantes redundancias terminológicas, tanto en el contenido de los distintos expedientes como en su nomenclatura y organismo emisor.

Un ejemplo de ello lo encontramos en la presencia de expedientes similares pero con distinta denominación, constituyendo así series distintas o paralelas, lo cual provocaba una gran confusión dentro de su gestión, como era el caso de la serie documental relativa a las "Ocupaciones".

Este tipo documental presentaba tres modalidades de Ocupación: Casas Forestales, Montes de Utilidad Pública y Vías Pecuarias; que en ocasiones se presentaban diferenciadas unas de otras y otras veces se agrupaban indistintamente, lo que evidenciaba la necesidad de normalización. Al mismo tiempo se presentaban innumerables tipos de "ocupaciones", entre los que se pueden destacar: Instalaciones de tuberías, Mejoras, Vallados, Pruebas deportivas, etc., cuya profusión había que controlar ya que aumentaban diariamente, provocando numerosos problemas en la gestión administrativa de la documentación porque las des- 


\begin{tabular}{|l|}
\multicolumn{1}{|c|}{ Concepto-OCUPACIÓN TERRENOS } \\
\hline Construcción balsa para riego \\
\hline Construcción balsón \\
\hline Construcción de embalse \\
\hline Construcción de embalse de agua \\
\hline Construcción de un embalse de agua \\
\hline Construcción de un embalse de plástico \\
\hline Construcción de un embalse de plástico \\
\hline Construcción embalse \\
\hline Construcción embalse de plástico \\
\hline Construcción embalse para riego \\
\hline Construcción embalse regulador para riego \\
\hline
\end{tabular}

Fig. 3 Cuadro de conceptos de ocupaciones

cripciones que se hacían en los propios expedientes resultaban ser de lo más variopintas.

Podemos tomar como ejemplo la descripción del concepto de una ocupación referente a la "construcción de embalse", se usaban descripciones tales como muestra el cuadro siguiente, pudiéndose unificar en un solo descriptor todas ellas, siendo el resto candidatas a figurar incluidas en nuestro lenguaje controlado como términos no descriptores (Fig. 3).

Hemos señalado anteriormente que otro de los problemas importantes de control terminológico se presentaba en las distintas nomenclaturas adoptadas por el organismo emisor de la documentación administrativa a lo largo de su historia. En este caso para describir un expediente de "Deslinde" que se realizó bajo la gerencia del Patrimonio Forestal del Estado y que aún sigue vigente, se tendría

\begin{tabular}{|l|}
\multicolumn{1}{c|}{ Organismo Emisor-DESLINDE } \\
\hline Patrimonio Forestal del Estado \\
\hline Instituto Nacional para la Conservación de la Naturaleza \\
\hline ICONA \\
\hline Agencia Regional para Medio Ambiente y la Naturaleza de la Región de Murcia \\
\hline ARMAN \\
\hline Dirección General del Medio Natural \\
\hline
\end{tabular}

Fig.4. Cuadro de las nomenclaturas del Organismo Emisor

Scire. $5: 2$ (jul.-dic. 1999) 115-130. 


\section{4}

que hacer referencia a las distintas denominaciones que tuvieron los gestores de dicho expediente (Fig. 4).

Todo esto presentaba un gran problema a la hora de informatizar las descripciones de este tipo de expedientes administrativos, ya que resaltaba la necesidad de normalización de terminología en dicha descripción. Si se establecía un cierto control sobre los términos utilizados se propiciaría una estandarización del proceso y una mayor eficiencia en la recuperación de la información tratada. Por ello, en el proceso de descripción del tipo documental "Deslindes", realizado

\section{DESLINDES}

\section{$N^{\circ}$ Monte:}

Nombre Monte:

TM:

Pertenencia:

Véase además-Pertenencia:

Orden Ministerial:

BOE:

BORM:

Notas:

Contenido Expediente:

Fig. 5. Ficha Normalizada para la descripción de expedientes de deslindes

siguiendo un formato de ficha normalizada se recogería el contenido de los campos que más tarde estaría reflejado en la base de datos relacional de Deslindes.

Dentro de la ficha normalizada, en el campo "Pertenencia" se utilizaron los descriptores correspondientes a la denominación actual del organismo emisor y en el campo "Véase además-Pertenencia" se establecerían las denominaciones antiguas, considerándose a éstas como "no descriptores", aumentando así el número de entradas de nuestro tesauro, enriqueciéndolo y garantizando así la recuperación de información por cualquiera de las antiguas denominaciones del Organismo Emisor de la Documentación Administrativa. 
Además dentro del campo "Nombre Monte" se establecían los nombres de los montes de utilidad pública establecidos como aceptados en el tesauro, aquellos nombres completos que figuraban en las Órdenes Ministeriales aprobatorias de los Deslindes, para evitar la pérdida de pertinencia en la recuperación de información y considerando la tendencia, dentro de la gestión técnica de este tipo de expedientes, a abreviar y reducir el nombre de los montes debido a la extensión de los mismos.

\subsection{Elaboración del Tesauro del Archivo Forestal.}

Si bien era importante el control de las series, aún lo era más el control de la terminología que en ellas se contenían, así como la descripción de las mismas, por ello se decidió elaborar una herramienta que permitiera la gestión y el control de los distintos expedientes administrativos que se encontraban en el Archivo Forestal, también los que se irían produciendo diariamente dentro del organismo emisor.

Entre las fuentes de información que se usaron para la realización del Tesauro del Archivo Forestal, se consultaron otros tesauros similares ya existentes, pero fueron fundamentalmente las fuentes personales las que más contribuyeron a la obtención del vocabulario, es decir a través de las distintas personas que generaban y gestionaban los distintos expedientes, se les fue preguntando sobre su trabajo y sus propuestas para la estructuración de dicho Tesauro, pasando desde los distintos administrativos, personal técnico y asesores jurídicos hasta los directivos del organismo emisor. Posteriormente realizamos una búsqueda en la base de datos ISOC con la idea de localizar propuestas previas en la misma línea que la nuestra. Del resultado de la misma, algo escaso por cierto, tuvimos acceso a los trabajos de Martín Suquía (1990) que redunda en la idea del control del vocabulario previo al desarrollo de una base de datos para un archivo. Realmente, la fuente de información más prolífica y productiva para nuestro propósito ha sido el propio Archivo, donde al tiempo que se procedía a su organización y tratamiento documental se iban efectuando las anotaciones pertinentes para completar el Tesauro. Así, fuimos seleccionando el léxico que debía incluirse en el lenguaje controlado. Para la adecuada elaboración del Tesauro se han seguido las especificaciones recogidas en la Norma UNE 50-106-90 (AENOR, 1990).

\section{Resultados.}

El Tesauro se estructura en seis campos semánticos, que detallamos a continuación:

Scire. 5 : 2 (jul.-dic. 1999) 115-130. 
1. Administración Pública: Este campo semántico recoge los términos sobre los distintos organismos públicos relacionados con la documentación tratada.

2. Expedientes Administrativos: Los términos referidos a los distintos tipos documentales y la variedad de los mismos se agrupan en este campo semántico.

3. Gestión Económica: Aquí se encuentran aquellos términos relacionados con la gestión económica del organismo emisor de la documentación archivística.

\section{EXPEDIENTES ADMINISTRATIVOS}

Expedientes de Propiedad

Amojonamientos

Montes Catalogados

Montes No Catalogados

Deslindes

Montes Catalogados

Montes No Catalogados

Permutas

Registros de Propiedad

Certificados de Colindancia

Expedientes de Vías Pecuarias

Tipos de Ocupaciones

Radiodifusión

Instalación de Antenas de

Instalación de Embalses Instalación de Líneas Eléctricas Instalación de Líneas Telefónicas Instalación de Parques Ecológicos Instalación de Postes Repetidores Instalación de Tuberías Mejoras y Embellecimientos Suministro Electrico Almacenes

Casas Goteros

Vallados 
4. Información y Documentación: Los términos sobre información y documentación relacionados con la documentación forestal se localizan en este campo.

5. Legislación: Recoge los términos sobre las normas y leyes vinculadas.

6. Tecnologías de la Información: Se agrupan los términos necesarios sobre Tecnologías de la Información que se relacionan con la documentación forestal.

Los formatos de presentación de nuestro Tesauro son: Sistemático, Alfabético y Permutado. La aplicación informática que se usó para la construcción del Tesauro fue el Sistema para la Gestión Automatizada de Tesauros, SGAT (7), bajo entorno Windows. Una vez finalizada la elaboración del Tesauro,

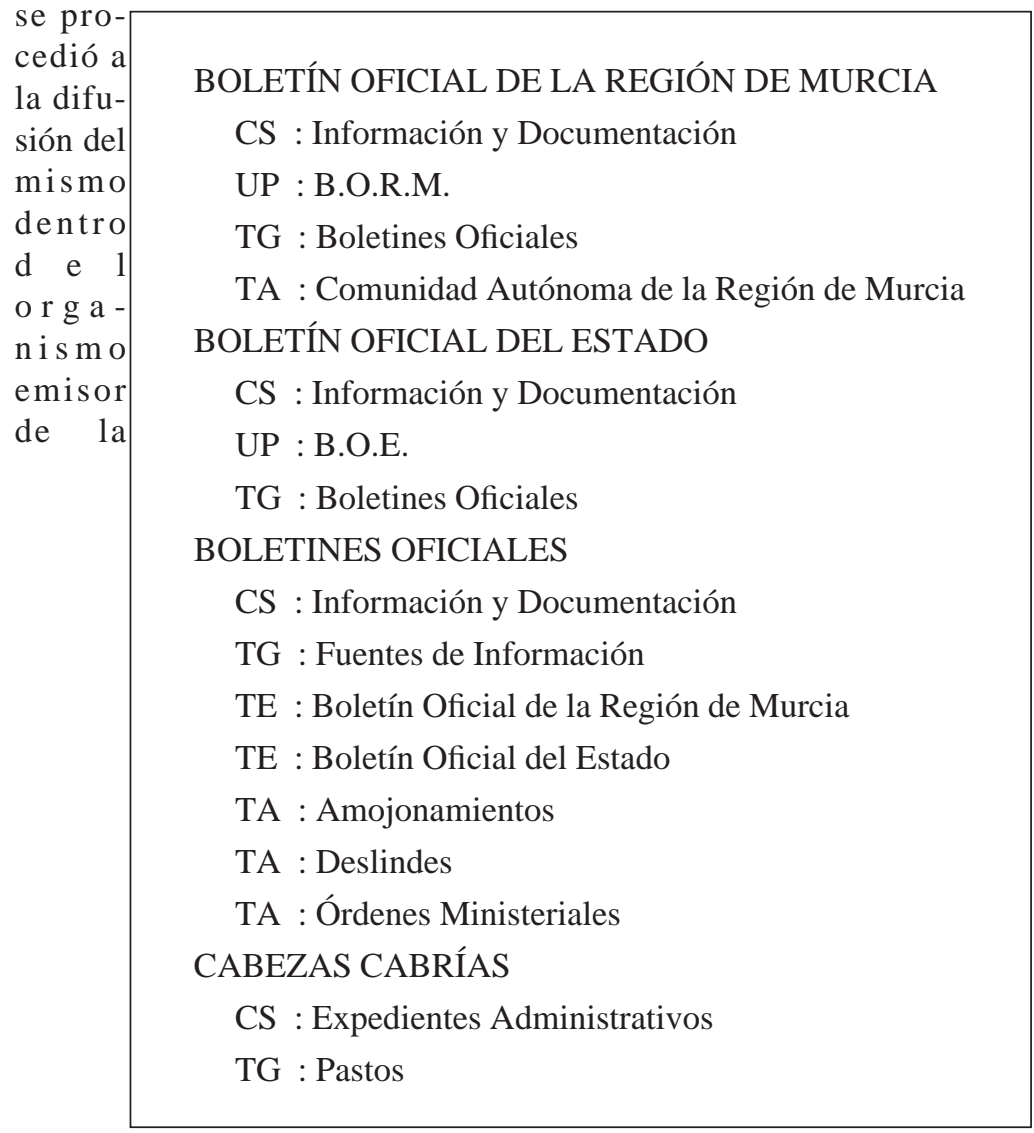

Fig. 7. Sección de la Presentación Alfabética del Tesauro del Archivo Forestal.

Scire. 5 : 2 (jul.-dic. 1999) 115-130. 
Documentación con el objetivo de que fuese utilizado en la génesis de las distintas series documentales, es decir en la gestión de la documentación administrativa, además de su uso dentro del Archivo Forestal.

El número total de términos que componen, en la actualidad, el Tesauro del Archivo Forestal es de 588, siendo 368 de ellos Descriptores y 214 No Descriptores. En cuanto a las relaciones semánticas establecidas entre los términos del Tesauro se presentan 121 relaciones de asociación, 363 relaciones de jerarquía y 214 relaciones de equivalencia.

\begin{tabular}{|c|c|}
\hline Símbolo & Relación \\
\hline Campo Semántico & CS \\
\hline Término Genérico & TG \\
\hline Término Específico & TE \\
\hline Término Asociado & TA \\
\hline Relaciones de Equivalencia & USE, UP \\
\hline Término Cabecera & TT \\
\hline
\end{tabular}

Fig. 8. Símbolos utilizados para describir las relaciones semánticas

Los símbolos que hemos utilizado para describir las relaciones semánticas establecidas entre los términos del Tesauro se recogen en la siguiente tabla (Fig. 8).

El Tesauro se encuentra sometido a permanente y continua actualización, ya que al lógico interés por refinar y apuntalar la estructura del mismo se une el hecho comentado en el apartado anterior de que el propio Archivo es la principal fuente proveedora de información para la recopilación de terminología, y como el tratamiento documental en el Archivo se ha constituido en un proceso prolongado, éste emana nuevos términos para su inclusión en el Tesauro de forma dinámica e interactiva. Van Slype (1991) indica que un Tesauro está destinado tanto a representar de manera unívoca el contenido de los documentos como de las consultas dentro de un sistema documental determinado y a ayudar al usuario en la indización de los documentos y de las consultas. Así, el Tesauro también tiene como objetivo incrementar el nivel de la recuperación de información, y en esta línea de trabajo hemos procedido a interrelacionar nuestro Tesauro mismo con la

Scire. $5: 2$ (jul.-dic. 1999) 115-130. 
implementación de un sistema de bases de datos en entorno relacional. Ideas en este sentido podemos encontrarlas en los trabajos de Rodríguez Muñoz (1990), Martínez Méndez (1992) y Asensi Artiga (1992), que apuestan por la propia gestión del Tesauro dentro del entorno de bases de datos relacionales y destacan su inherente trascendencia en los procesos de recuperación de la información.

En nuestro caso, se ha procedido al diseño de una serie de bases de datos en las que se incluiría, junto a la serie de campos destinados a la descripción del fondo documental, un campo que denominaremos genéricamente DESCRIPTOR, el cual tendría limitada la entrada al vocabulario controlado manejado en nuestro Tesauro, para así intentar alcanzar esa normalización que en un principio se pretendía y conseguir también que la recuperación de información en las bases de datos sea lo más precisa posible. Las bases de datos que se han desarrollado son: DESLINDE-AMOJONAMIENTOS, OCUPACIONES, FINCAS, DENUNCIAS FORESTALES, CONTRATACIONES TEMPORALES $\mathrm{e}$ INFORMACIÓN BIBLIOGRÁFICA, integradas todas ellas dentro del sistema gestor relacional Access de Microsoft. Aunque estas bases de datos se presentan de forma individualizadas, están diseñadas para ser integradas e interrelacionadas unas con otras cuando sea necesario. Esto es posible por el uso de una tabla que hace de unión entre todas ellas y que se establece como el pilar de toda la estructura relacional. Esta tabla es la denominada "Montes", en la que se recogen los datos identificativos de todos los montes de utilidad pública, tanto los catalogados como los no catalogados, pertenecientes a la Comunidad Autónoma de la Región de Murcia.

\section{Conclusiones}

Con el desarrollo de este trabajo creemos haber destacado suficientemente la importancia de la presencia de un Tesauro para facilitar una adecuada gestión documental en un archivo administrativo, contribuyendo a la optimación de las funciones desempeñadas en el seno del organismo productor y por ende, favoreciendo un incremento en la calidad de dicha gestión.

También creemos interesante señalar la necesidad de automatización de los archivos existentes en las distintas Administraciones Públicas ya que si entendemos al Archivo como una unidad informativa activa, la información debe estar dispuesta de forma que su recuperación sea pertinente en tiempo y forma. Además de resaltar la importancia de la figura del Gestor de Información y Documentación Administrativa en este entorno.

En esta línea, sorprende la escasa o nula presencia de este tipo de profesional de la información en el seno de las Administraciones Públicas, situación que pensamos debe variar sustancialmente en pos de la modernización de estas institu-

Scire. 5 : 2 (jul.-dic. 1999) 115-130. 
ciones, más si cabe cuando otras administraciones si están desarrollando actividades en otras líneas de trabajo.

En un nivel más local, y centrándonos en el caso particular de la Consejería de Medio Ambiente, Agricultura y Agua, postulamos por la creación de un sistema integrado de gestión de información y documentación administrativa, que complemente la actividad que se viene desarrollando en los Archivos.

\section{Referencias Bibliográficas.}

Asensi Artiga, V. ; et al. (1992). Implementación de un módulo de gestión de tesauros en un sistema gestor documental. // Actas de las 4es Jornades Catalanes de Documentació. Barcelona : SOCADI, 1992.

Asociación española de normalización y certificación (1990). UNE 50-106-90 : Documentación : Directrices para el establecimiento y desarrollo de tesauros monolingües. Madrid: AENOR, 1990.Office for government online. The Architecture of Access to Information Resources: Chapter 6. Disponible en URL: <http://www.ogit.gov.au/publications/imsc/chapter6.htm>.

Government Information Locator Service (GILS). Disponible en URL: <http://www.ahcpr.gov/gilsinfo.htm>.

Martín Suquía, R. (1990). El control del lenguaje en el proceso de formación de una base de datos de archivo: IRARGI 1. // Actas de las III Jornadas Españolas de Documentación Automatizada. Palma de Mallorca: FESABID, 1990.

Martínez Méndez, F. J. ; et al. (1992). Diseño lógico-conceptual de tesauros. // Actas de las 4es Jornades Catalanes de Documentació. Barcelona: SOCADI, 1992.

Parada, R. (1993). Derecho Administrativo III: Bienes públicos. Derecho Urbanístico. $5^{\text {a }}$ ed. Madrid: Marcial Pons, 1993.

Pastor Sánchez, J.A. ; Saorín Pérez, T. (1996). Un interfaz de hipertexto para un sistema de gestión de tesauros. // Scire. $2: 1$ (en.-jun. 1996) 51-62.

Rodríguez Muñoz, J. V. ; et al. (1990). Los modelos de datos como alternativa en la construcción de tesauros. // Actas de las III Jornadas Españolas de Documentación Automatizada. Palma de Mallorca : FESABID, 1990.

Van Slype, G. (1991). Los Lenguajes de Indización : Concepción, construcción y utilización en los sistemas documentales. Salamanca : Fundación Germán Sánchez Ruipérez, 1991.

What is GILS? : Government Information Locator Service $=$ Service de localisation de l'information du government. Disponible en URL: <http://gils.srv.gc.ca/english/gils_info/what_is_gils.cfm〉. 